\title{
Can Radiographs Predict the Use of Modular Stems in Developmental Dysplasia of the Hip?
}

\author{
Christopher L. Peters MD, Jesse Chrastil MD, \\ Gregory J. Stoddard MPH, MBA, Jill A. Erickson PA-C, \\ Mike B. Anderson MSc, Christopher E. Pelt MD
}

Published online: 17 July 2015

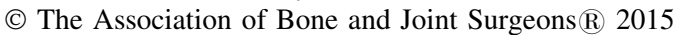

\begin{abstract}
Background Abnormal anatomy frequently results in the use of a modular stem in patients undergoing primary total hip arthroplasty (THA) for developmental dysplasia of the hip (DDH). However, because these stems are not always available in the operating room, it would be helpful if standard radiographic views could be analyzed in such a way that patients whose femoral anatomy might call for stem modularity could be anticipated before surgery. To our knowledge, no such parameters have been defined.

Questions/purposes In the senior author's practice, we used femoral neck anteversion of more than $25^{\circ}$ as a determinant for use of a modular stem. Given this criterion, we asked if we could reliably identify plain film
\end{abstract}

One the authors certify that he (CLP), or a member of his immediate family, has or may receive payments or benefits, during the study period, an amount of USD 100,001 to USD 1,000,000 from Biomet, Inc (Warsaw, IN, USA). One the authors certify that he (CEP), or a member of his immediate family, has or may receive payments or benefits, during the study period, an amount of USD 10,000 to USD 100,000 from Biomet, Inc (Warsaw, IN, USA).

All ICMJE Conflict of Interest Forms for authors and Clinical Orthopaedics and Related Research ${ }^{\mathbb{R}}$ editors and board members are on file with the publication and can be viewed on request. Clinical Orthopaedics and Related Research ${ }^{\mathbb{R}}$ neither advocates nor endorses the use of any treatment, drug, or device. Readers are encouraged to always seek additional information, including FDAapproval status, of any drug or device prior to clinical use.

Each author certifies that his or her institution approved or waived approval for the reporting of this case and that all investigations were conducted in conformity with ethical principles of research.

C. L. Peters ( $₫)$, J. Chrastil, G. J. Stoddard, J. A. Erickson, M. B. Anderson, C. E. Pelt

Department of Orthopaedics, University of Utah, 590 Wakara

Way, Salt Lake City, UT 84108, USA

e-mail: chris.peters@hsc.utah.edu radiographic parameters of the femur that predict the use of modular stems. We looked at the following: (1) the neckshaft angle based on the anteroposterior (AP) radiograph (alpha); (2) the neck-shaft angle from the crosstable lateral radiograph (beta); and (3) the calculated femoral anteversion angle.

Methods We reviewed preoperative radiographs from 50 of 67 patients (79 hips) who had a primary diagnosis of DDH and underwent primary THA from January 1999 to February 2007 inclusive. Hips with prior femoral-sided surgery $(n=2)$ or without preoperative films $(n=19)$ were excluded. Furthermore, patients with bilateral hips had the second hip excluded $(n=8)$. Twenty-one of 50 received a modular femoral stem based on the criterion of intraoperative neck-shaft anteversion of greater than $25^{\circ}$ as measured by the senior surgeon (CLP), whereas the remainder received tapered nonmodular stems. There were no differences in age, sex, height, or weight between the modular stem group and tapered stem group. Radiographs were evaluated to record the parameters listed.

Results Patients in whom modular femoral stems were used had a greater mean AP (alpha) neck-shaft angle compared with patients who received tapered nonmodular stem $\left(152^{\circ} ; 95 \%\right.$ confidence interval $[\mathrm{CI}], 146^{\circ}-157^{\circ}$ versus $\left.137^{\circ} ; 95 \% \mathrm{CI}, 134^{\circ}-141^{\circ} ; \mathrm{p}<0.001\right)$ with an optimal cutoff point for determining the use of modular stems of $\geq$ $142^{\circ}$ (receiver operating characteristic [ROC] area $=73 \%$ ). Hips in which modular femoral stems were chosen had a smaller mean lateral (beta) neck-shaft angle $\left(152^{\circ} ; 95 \% \mathrm{CI}\right.$, $148^{\circ}-157^{\circ}$ versus $161^{\circ} ; 95 \% \mathrm{CI}, 158^{\circ}-164^{\circ} ; \mathrm{p}=0.003$ ) with an optimal cutoff point of $\leq 153^{\circ}(\mathrm{ROC}$ area $=65 \%)$. Hips in which modular femoral stems were used had a higher femoral anteversion angle (mean $45^{\circ}$; $95 \%$ CI, $37^{\circ}$ $54^{\circ}$ versus $21^{\circ}$; interquartile range, $17^{\circ}-25^{\circ} ; \mathrm{p}<0.001$ ) with an optimal cutoff of $\geq 32^{\circ}$ (ROC area $=80 \%$ ). 
Conclusions Preoperative radiographs anticipated the use of modular stems during THA for DDH in a practice where modular stems were chosen on the basis of a neck-shaft angle of greater than $25^{\circ}$ measured at surgery. We found that this could be predicted on preoperative radiographs based on smaller lateral neck-shaft angles, steeper AP radiographic neck-shaft angles, and increased femoral anteversion calculated using these angles. Prospective studies are needed to better determine if these cutoff values adequately predict the use of modular stems.

\section{Introduction}

Developmental dysplasia of the hip (DDH) is characterized by abnormal acetabular and femoral morphologies and is thought to be one of the leading causes of secondary osteoarthritis resulting in THA [3]. The osseous anatomy associated with hip dysplasia makes these reconstructions more difficult. The acetabulum often is shallow with superior or anterior bone loss. The femur often is excessively anteverted and valgus. The medullary canal can be small and abnormally shaped with a smaller mediolateral diameter than AP diameter [8, 12]. Because of these associated anatomic variants, some authors recommend the use of modular or specially designed components to accommodate the shape of the dysplastic femur, whereas other authors have published good long-term results with cementless tapered stems [4, 8].

Biant et al. [1] and Tamegai et al. [13] have both reported excellent results in patients with $\mathrm{DDH}$ who underwent THA with a modular femoral stem. The benefits of using the modular system include the ability to adjust femoral anteversion allowing for more optimal placement of the prosthesis as well as functional advantages of reliable fixation and the prevention of dislocations postoperatively $[1,13]$. Unfortunately, these stems are not always readily available in the operating room, and it would be helpful if standard radiographic views could predict before surgery which patients would be likely to benefit from their use. Three-dimensional (3-D) CT scans allow for accurate assessments of anteversion but are timeconsuming, costly, and require substantial radiation. To our knowledge, no parameters have been defined using plain radiographs that can help the surgeon anticipate the use of a modular stem during THA for DDH.

We therefore asked if we could reliably identify preoperative plain film radiographic parameters of the femur that predicted the use of modular stems during THAs for $\mathrm{DDH}$. The general indication for using these stems was femoral anteversion of more than $25^{\circ}$ measured at the time of surgery. In this retrospective study, we looked at the following: (1) the neck-shaft angle based on the AP radiograph (alpha); (2) the neck-shaft angle from the lateral radiograph (beta); and (3) the femoral anteversion angle as calculated from the prior two angles.

\section{Materials and Methods}

After we received an exemption from the institutional review board for secondary data analysis, we performed a retrospective search of our surgical database. We identified 630 primary THAs that were performed by the senior author (CLP) between February 1999 and January 2007 inclusive. From these, we identified 79 hips in 67 patients who had a primary diagnosis of DDH as determined by lateral center-edge angle $<20^{\circ}$ and an upwardly sloping acetabular sourcil. Hips were excluded if they had a history of prior femoral-sided hip surgery $(n=2)$ or did not have digital preoperative films available $(n=19)$. This resulted in a series of 58 hips in 50 patients. To avoid potential bias related to bilateral hips and implant selection, where the surgeon might use a modular stem in the second hip because it was used in the first, the first hip that underwent THA was selected for the review resulting in 50 patients with 50 hips. We assessed operative reports to identify the type of implant used and the rationale for the implant. S-ROM modular femoral stems (DePuy Synthes, Warsaw, IN, USA) were placed in 21 of 50; the remaining 29 patients received a tapered nonmodular stem (Biomet Orthopaedics, Warsaw, IN, USA). This stem has a moderate metaphyseal flare that does not accommodate excessive anteversion (Fig. 1). In this single-surgeon experience, the rationale for femoral stem selection was principally based on femoral anteversion: if femoral anteversion was estimated to be greater than $25^{\circ}$ by the senior surgeon during surgery, a modular stem was typically selected. There were no differences in patient age, height, weight, or sex between the modular stem (MS) group and tapered stem (TS) group (Table 1).

To evaluate the radiographic characteristics that predicted the surgeon's selection of MS versus TS, we performed a radiographic analysis of the 50 hips. Lateral center-edge angle [14] as well as Crowe's classifications [3] and the Hartofilakidis classification [15] were used to confirm a diagnosis of dysplasia on preoperative films. The angles were obtained from a described radiographic method [9]. The MS group demonstrated smaller lateral center-edge angles (mean $9^{\circ} ; 95 \%$ confidence interval [CI], $3^{\circ}-15^{\circ}$ versus mean $\left.19^{\circ}, 95 \% \mathrm{CI}, 15^{\circ}-22^{\circ} ; \mathrm{p}=0.003\right)$. Modular stems were found to have a slightly higher classification using Crowe's classification (median 1.5; interquartile range [IQR], 1-2 versus median 1; IQR, 1-1; $\mathrm{p}=0.006)$. This was also the case for the Hartofilakidis classification where MS stems had a median classification 


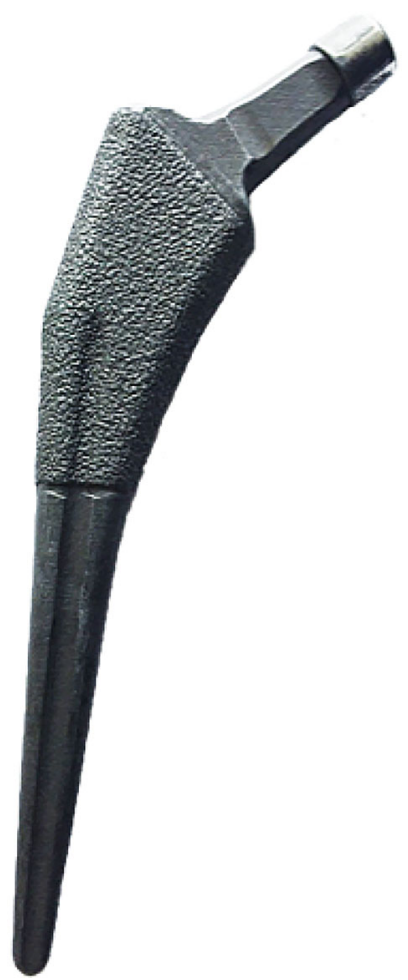

Fig. 1 Tapered nonmodular stem is shown (Biomet Orthopaedics, Warsaw, IN, USA).

Table 1. Demographic comparison between stem types

\begin{tabular}{lccc}
\hline Demographic & Modular & Tapered & $\mathrm{p}$ value \\
\hline Age (years), mean (SD) & $40(10)$ & $46(12)$ & 0.09 \\
Height (inches), mean (SD) & $65(3)$ & $65(3)$ & 0.99 \\
Weight (pounds), mean (SD) & $174(57)$ & $174(52)$ & 0.99 \\
Sex (number) & & & \\
Male & $6(29 \%)$ & $6(21 \%)$ & 0.74 \\
Female & $15(71 \%)$ & $23(79 \%)$ & \\
\hline
\end{tabular}

of 2 (IQR, 2-2) compared with 1 (IQR, 1-2) in the TS group ( $\mathrm{p}=0.001)$.

Clinically, our radiographs have been standardized to ensure reproducibility. The crosstable lateral radiograph is obtained with the patient supine on the radiograph table. The contralateral hip and knee are flexed up out of the field of the hip being imaged. The extremity of interest is internally rotated $15^{\circ}$ to expose the full anterolateral and posterolateral femoral head-neck junction. The radiograph tube is orientated parallel to the table at a $45^{\circ}$ angle to the imaged limb and centered on the femoral head. The AP radiograph is obtained with the patient in the standing position with lower extremities oriented in $15^{\circ}$ of internal rotation to maximize the femoral neck length. The distance from the imaging tube to the plate is approximately $120 \mathrm{~cm}$ in a perpendicular orientation. The image is centered

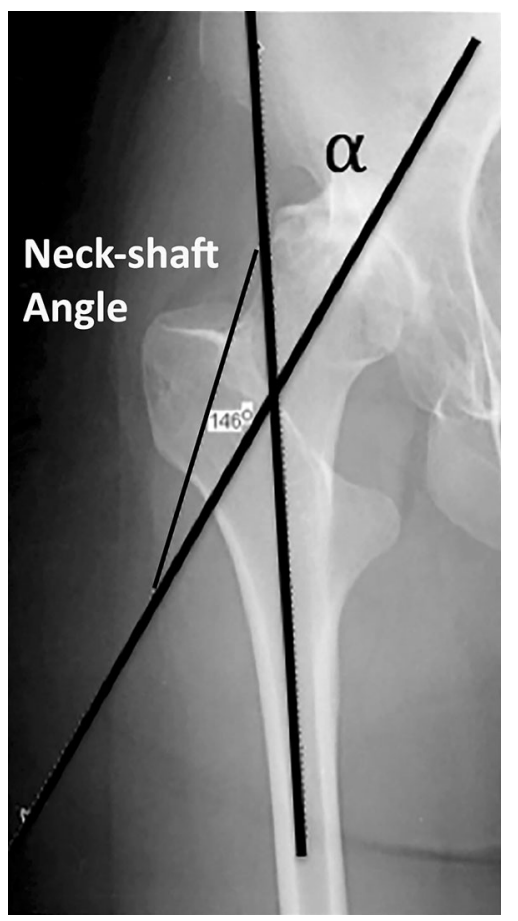

Fig. 2 This figure demonstrates the supplementary neck-shaft angle of Ogata and Goldsand's $\alpha$ angle.

between the superior border of the pubic symphysis and a line drawn connecting the anterosuperior iliac spines to visualize the entire bony pelvis and to minimize scatter and parallax effect. This standardization assures true anatomy and minimized a rotational misconstruction.

Radiographic measurements were performed using our digital PACs system (iSite v3.6; Philips Healthcare, Andover, MA, USA) by two experienced clinicians (JC, JAE) on preoperative films. The AP radiograph was used to obtain the alpha neck-shaft angle (Fig. 2) and the crosstable lateral radiograph was used to measure the beta neckshaft angle (Fig. 3). The initial reader (JC) performed all reads from preoperative films, but may have visualized the stem in the PACS system. Similarly, a second, experienced clinician (JAE) then read the same radiographs twice (blinded to the first reads), 1 week apart, to allow for assessment of interrater and intrarater reliability and agreement. The concordance correlation coefficient $\left(r_{c}\right)$ and a Bland-Altman analysis were used for this analysis [2, 6]. The amount of agreement between readers for the $r_{c}$ was classified as minimal, $<0.2$; poor, 0.2 to $<0.4$; moderate, 0.4 to $<0.6$; strong, 0.6 to $\leq 0.8$; and almost perfect, $>0.8$ [10]. There was strong agreement between the two clinicians when measuring the alpha neck-shaft angle and the beta neck-shaft angle (Table 2). Furthermore, the intrarater agreement also demonstrated almost perfect agreement between reads (Table 3 ). Thus, the measurements from the first reader were used in this analysis. 
Based on these measurements, we then calculated the patient femoral anteversion based on the formula presented by Ogata and Goldsand [9] $(\tan \theta=\tan \beta / \tan \alpha)$. In their original publication, they described $\alpha$ (Fig. 2) and $\beta$ (Fig. 3) as supplementary angles to what we calculated as neck-shaft angles, which we then corrected for in our equations. We used Microsoft Excel (Microsoft Inc, Redmond, WA, USA) to perform the calculations as follows:

1) Equation 1: $180^{\circ}$ - AP neck-shaft angle $=\alpha$

2) Equation 2: $180^{\circ}$ - lateral neck-shaft angle $=\beta$

3) Equation 3: " $\theta=$ TAN (RADIANS $[\beta]$ )/TAN (RADIANS [ $\alpha]$ )"

4) Equation 4: "Anteversion = DEGREES(ATAN $[\theta]$ )

Ordinal data were compared using a Wilcoxon MannWhitney test. Additionally, an independent samples t-test was used to compare radiographic measures between the groups. Spearman's rank correlation $\left(\mathrm{r}_{\mathrm{s}}\right)$ was used to assess the relationship between the actual angle measured and the

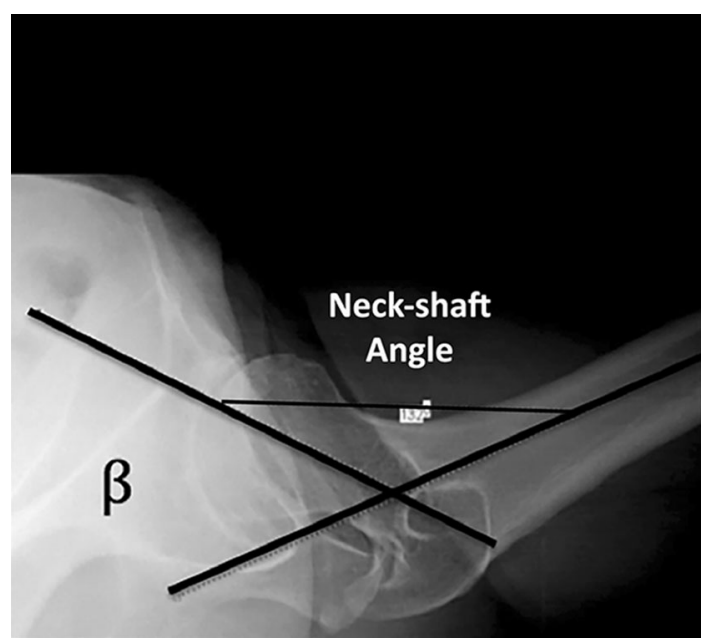

Fig. 3 This figure demonstrates the supplementary neck-shaft angle of Ogata and Goldsand's $\beta$ angle. intraoperative stem decision. Correlation coefficients were assessed as previously mentioned. Furthermore, we dichotomized the beta neck-shaft angle at every possible cutoff point and computed the area under the receiver operating characteristic curve (ROC area) using the dichotomized variable as a binary diagnostic test for the outcome. This was repeated for every value of the variable. The cutoff point that resulted in the maximum ROC area was identified as the optimal cutoff point. To verify the stability of the optimal cutoff point, meaning that cutoff point would be selected as the optimal cutoff point in the future, we used an algorithm adapted from Royston and Sauerbrie [11]. The algorithm is as follows: (1) draw a bootstrap resample of size, the original sample size; (2) compute the ROC area for every possible cutpoint in the resample; (3) record which cutoff point optimized the ROC area; (4) repeat steps 1 through 31000 times; (5) summarize the frequency of the cutoff points that optimized the ROC area; and (6) select the cutpoint with the highest frequency percent as being the most stable cutpoint.

After the optimal cutpoint was selected, we then performed an internal bootstrap validation of the cutpoint's test characteristics (sensitivity, specificity, and ROC area) using Harrell et al.'s [5] algorithm. The test characteristics for any prediction equation, or optimal cutoff point, are known to always be greater in the sample it was derived on. The internal bootstrap validation subtracts out this overfitting, or optimism, to provide test characteristic values and CIs that can be expected in future samples or future patients.

\section{Results}

The cutoff point for the AP neck-shaft angle that best predicted the use of a modular stem in patients undergoing THA for DDH was $\geq 142^{\circ}$. On evaluation of the

Table 2. Interrater agreement on alpha and beta neck-shaft angles

\begin{tabular}{llllll}
\hline Angle & $\mathrm{r}_{\mathrm{c}}$ & $95 \% \mathrm{CI}$ & Bland-Altman average difference (SD) & $95 \%$ limits of agreement & Pearson's $\mathrm{r}$ \\
\hline Alpha neck-shaft & 0.81 & $0.71-0.90$ & $-4.12(7.34)$ & -19 to 10 & 0.85 \\
Beta neck-shaft & 0.84 & $0.75-0.93$ & $-0.56(5.6)$ & -12 to 10 & 0.84 \\
\hline
\end{tabular}

$\mathrm{r}_{\mathrm{c}}=$ correlation coefficient; $\mathrm{CI}=$ confidence interval.

Table 3. Intrarater agreement on alpha and beta neck-shaft angles measured on a subsample (first 25 patient) 1 week apart from each other and blinded to prior results

\begin{tabular}{lllllc}
\hline Angle & $r_{c}$ & $95 \%$ CI & $\begin{array}{l}\text { Bland-Altman average } \\
\text { difference (SD) }\end{array}$ & $\begin{array}{l}\text { 95\% limits } \\
\text { of agreement }\end{array}$ \\
\hline Alpha neck-shaft & 0.95 & $0.91-0.99$ & $-1.0(4.1)$ & -9 to 7 & 0.95 \\
Beta neck-shaft & 0.89 & $0.79-0.99$ & $1.05(3.95)$ & -6.7 to 8.8 & 0.90 \\
\hline
\end{tabular}

$\mathrm{r}_{\mathrm{c}}=$ correlation coefficient; $\mathrm{CI}=$ confidence interval. 
Table 4. Test characteristics

\begin{tabular}{|c|c|c|c|c|c|}
\hline \multirow[t]{2}{*}{ Angle } & \multicolumn{2}{|c|}{ Cutpoints for modular stem decision } & \multicolumn{3}{|c|}{ Test characteristics $(\%)(95 \%$ confidence intervals) } \\
\hline & Positive & Negative & & Observed & Optimism corrected* \\
\hline \multirow[t]{3}{*}{ Alpha } & \multirow[t]{3}{*}{$\geq 142$} & \multirow[t]{3}{*}{$<142$} & Sensitivity & $81(58-95)$ & $77(54-91)$ \\
\hline & & & Specificity & $72(53-87)$ & $69(49-84)$ \\
\hline & & & $\mathrm{ROC}$ area & $77(65-89)$ & $73(61-85)$ \\
\hline \multirow[t]{3}{*}{ Beta } & \multirow[t]{3}{*}{$\leq 153$} & \multirow[t]{3}{*}{$>153$} & Sensitivity & $57(34-78)$ & $51(28-72)$ \\
\hline & & & Specificity & $83(64-94)$ & $80(61-91)$ \\
\hline & & & $\mathrm{ROC}$ area & $70(57-83)$ & $65(52-78)$ \\
\hline \multirow[t]{3}{*}{ Anteversion } & \multirow[t]{3}{*}{$\geq 32$} & \multirow[t]{3}{*}{$<32$} & Sensitivity & $85(62-97)$ & $78(55-91)$ \\
\hline & & & Specificity & $86(68-96)$ & $83(65-93)$ \\
\hline & & & ROC area & $86(75-96)$ & $80(70-91)$ \\
\hline
\end{tabular}

* From internal bootstrap validation, test characteristic performance that can be expected in future patients; ROC = receiver operating characteristic.

Table 5. Decisions based on the three cutpoints

\begin{tabular}{lll}
\hline & \multicolumn{2}{l}{ Stem selected by surgeon } \\
\cline { 2 - 3 } $\begin{array}{l}\text { Angle decisions: } \\
\text { beta }\end{array}$ & $\begin{array}{l}\text { Tapered stem } \\
\text { (number) }\end{array}$ & $\begin{array}{l}\text { Modular stem } \\
\text { (number) }\end{array}$ \\
alpha & & \\
anteversion & 1 & 10 \\
\hline+++ & 1 & 6 \\
-++ & 2 & 1 \\
+-+ & 2 & 1 \\
+- & 6 & 1 \\
-+- & 17 & 2 \\
--- & &
\end{tabular}

$+=$ test positive (met cutoff rule for deciding on modular stem); $-=$ test negative (did not meet cutoff rule); for example, $-++=$ negative decision based on beta angle, positive decision based on alpha angle, and positive decision based on anteversion.

preoperative radiographs, the MS group had a greater mean AP radiographic neck-shaft angle compared with the TS group $\left(152^{\circ} ; 95 \% \mathrm{CI}, 146^{\circ}-157^{\circ}\right.$ versus $137^{\circ} ; 95 \% \mathrm{CI}, 134^{\circ}-$ $\left.141^{\circ} ; \mathrm{p}<0.001\right)$. There was a moderate agreement between the mean AP neck-shaft angle and the stem decision $\left(\mathrm{r}_{\mathrm{s}}=\right.$ $0.54 ; 95 \%$ CI, 0.31-0.71; $\mathrm{p}<0.001)$. The cutoff point was verified to be stable in a bootstrap simulation. In 1000 bootstrap resamples, which mimic future patient data sets, the cutpoint of $\geq 142^{\circ}$ was found in $54 \%$ of the resamples. The next most frequent optimal cutoff point was $145^{\circ}$, which occurred in only $11 \%$ so that $\geq 142^{\circ}$ was the clearcut most reliable cutoff. The decision rule of $\geq 142^{\circ}$ to require a modular stem has validated sensitivity of $77 \%(95 \% \mathrm{CI}$, $54 \%-91 \%)$, specificity of $69 \%$ (95\% CI, 49\%-84\%), and ROC area of $73 \%$ (95\% CI, 61\%-85\%) (Table 4).

The cutoff point for the lateral neck-shaft angle that best predicted the use of a modular stem was $\leq 153^{\circ}$. The MS group had a smaller mean lateral neck-shaft angle $\left(152^{\circ}\right.$; $95 \% \mathrm{CI}, 148^{\circ}-157^{\circ}$ versus $161^{\circ}$; $95 \% \mathrm{CI}, 158^{\circ}-164^{\circ} ; \mathrm{p}=$ $0.003)$. There was a moderate negative correlation with the lateral neck-shaft angle and the intraoperative stem choice $\left(r_{\mathrm{s}}=-0.41 ; 95 \% \mathrm{CI},-0.62\right.$ to $\left.-0.15 ; \mathrm{p}=0.003\right)$. In 1000 bootstrap resamples, which mimic future patient data sets, the cutoff point of $\leq 153^{\circ}$ was found in $32 \%$ of the resamples. The next most frequent optimal cutoff point was $\leq 162^{\circ}$, which occurred in only $20 \%$ so that $\leq 153^{\circ}$ was the clearcut most reliable cutoff point. The decision rule of $\leq 153^{\circ}$ to require a modular stem has validated sensitivity of $51 \%(95 \%$ CI, $28 \%-72 \%)$, specificity of $80 \%$ (95\% CI, $61 \%-91 \%)$, and ROC area of $65 \%(95 \%$ CI, 52\%-78\%) (Table 4).

The cutoff point for femoral anteversion that best predicted the use of a modular stem was $\geq 32^{\circ}$. Femoral anteversion, as calculated with our modification of Ogata's original equation [9], revealed that the MS group had a higher femoral anteversion angle (mean $45^{\circ}$; $95 \%$ CI, $37^{\circ}$ $54^{\circ}$ versus $21^{\circ}$; IQR, $\left.17^{\circ}-25^{\circ} ; \mathrm{p}<0.001\right)$. There was a strong correlation between the estimated femoral anteversion and the intraoperative stem choice $\left(r_{s}=0.67 ; 95 \% \mathrm{CI}\right.$, $0.47-0.80 ; \mathrm{p}<0.001)$. This cutoff point was verified to be stable in a bootstrap simulation. In 1000 bootstrap resamples, which mimic future patient data sets, the cutoff point of $\geq 32^{\circ}$ was found in $35 \%$ of the resamples. The next most frequent optimal cutoff point was $27^{\circ}$, which occurred in only $14 \%$ so that $\geq 32^{\circ}$ was the clearcut most reliable cutoff point. The decision rule of $\geq 32^{\circ}$ to require a modular stem has validated sensitivity of $78 \%(95 \% \mathrm{CI}$, $55 \%-91 \%)$, specificity of $83 \%$ (95\% CI, 65\%-93\%), and ROC area of $80 \%$ (95\% CI, 70\%-91\%) (Table 4).

These cutoff predictions (Fig. 3) confirm the accuracy of the alpha neck-shaft angle and the anteversion angle in identifying patients who received modular stems. Exceptions to the predictive cutoff values, however, were seen 
(Table 5). One patient who received a tapered stem, yet had met all three cutoff definitions for a modular stem, had angles as follows: lateral neck-shaft angle $=151^{\circ}, \mathrm{AP}$ neck-shaft angle $=155^{\circ}$, and an anteversion angle of $50^{\circ}$. Two patients who received a modular stem, yet had met none of the cutoff definitions, had angles as follows: lateral neck-shaft angle $=160^{\circ}$, AP neck-shaft angle $=140^{\circ}$, and anteversion $=23^{\circ}$ and lateral neck-shaft angle $=162^{\circ}$, AP neck-shaft angle $=131^{\circ}$, and anteversion $=16^{\circ}$.

\section{Discussion}

The dysplastic femur poses a challenge for adult reconstructive surgeons during THA attributable primarily to altered morphology, particularly on the femoral side. Femoral morphology includes an extensively anteverted and valgus femur as well as a small and misshapen medullary canal. Excellent results have been reported with the use of modular stems during THA in these challenging dysplastic hips [1, 13]; however, these stems are not always available in the operating room. The availability and ease of obtaining preoperative radiographs makes them an optimal choice for determining the need for modular stems in preoperative planning. We found that by using neckshaft angles obtained from preoperative crosstable lateral and AP radiographs and calculating anteversion, from previously published formulas, allowed us to predict the use of modular stems [9].

There are several limitations to this study. First, it is a retrospective chart and radiographic review of a singlesurgeon series of patients with hip dysplasia who underwent THA with either a tapered nonmodular stem or a modular stem that relies on proximal metaphyseal ingrowth. Thus, these results might not be applicable to all practices. For example, Faldini et al. [4] reported on a series of 46 patients who underwent primary THA secondary to DDH using a Wagner uncemented stem aimed at achieving $10^{\circ}$ to $20^{\circ}$ of anteversion. However, they did not report on how many patients achieved that alignment. Regardless, they note excellent osteointegration and remarked that all patients were satisfied with both function and pain relief. One caveat to the use of a Wagner conelike stem is that anteversion is associated with a loss of femoral offset, which occasionally can be problematic. The Wagner stem does not account for this, but the SROM modular stem has the ability to address both anteversion and offset. Second, there is potential for inaccurate and potentially unreproducible measures obtained intraoperatively. The choice of femoral component was determined by the judgment of the senior author (CLP) intraoperatively and femoral anteversion was not quantified in operative reports. Although the intraoperative decision for the use of
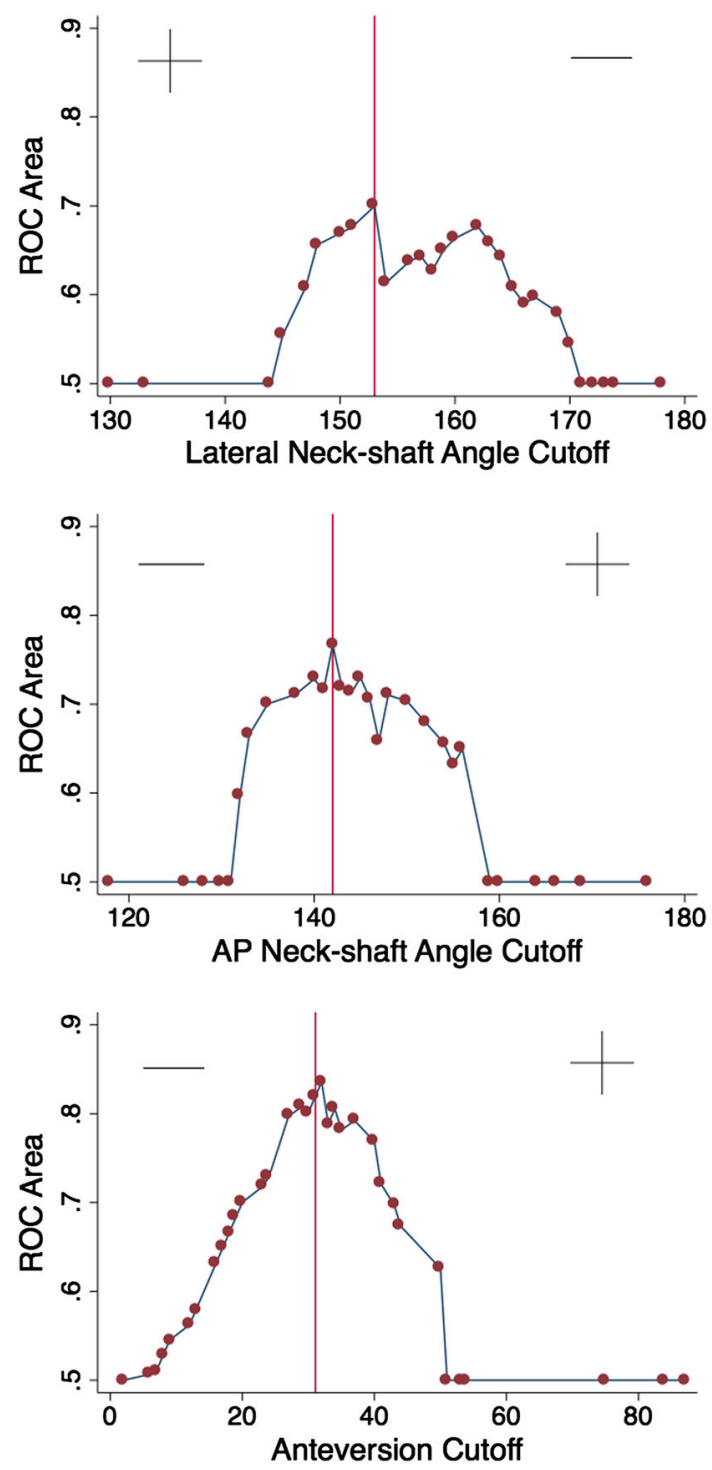

Fig. 4 The line graphs demonstrate the optimal cutoff points of each angle for stem decision with + representing modular stem selection and - representing tapered stem selection.

a modular femoral stem was based on femoral anteversion being approximately $25^{\circ}$, we had inadequate records to verify this. However, even if we routinely measured and recorded this, we still would not have an optimal cutoff point to reference, hence the need for this study. Furthermore, because the cohort was assembled over a long time period, the possibilities of changes in surgical technique are not controlled. However, with regard to the technique to address increased femoral anteversion, the surgeons' intraoperative choice of using a modular stem when femoral anteversion exceeded approximately $25^{\circ}$ was consistent throughout the study period. Although the statistical methodology of the radiographic analysis was independent of this and the question of the study was not dependent on intraoperative quantification of femoral 
anteversion, the stem decision was. Third, the two groups were not similar in grading or classification of DDH. However, this is in fact somewhat reassuring, because the level of femoral dysplasia agrees with the level of acetabular dysplasia. Finally, the cutoff points cannot be claimed to be the absolute best. A surgeon might consider being close to the cutoff points (Fig. 4), the peaks, or near the cutoff points, the plateaus, as a decision rule, rather than the exact values reported after observing the patient both clinically and within the operating room.

We developed decision rules based on preoperative radiographs that should predict the intraoperative choice of a modular stem when that choice is made based on an estimated intraoperative anteversion of $>25^{\circ}$; the best cutoff values on preoperative radiographs were AP neckshaft angle of $\geq 142^{\circ}$, lateral neck-shaft angle of $\leq 153^{\circ}$, and femoral anteversion of $\geq 32^{\circ}$. To the best of our knowledge, there are no previous studies investigating whether plain radiographs can predict the need for a modular stem in dysplastic femora. Our findings do seem consistent with observations of Noble et al., [8] who investigated the 3-D shape of the dysplastic femur and found that the primary deformity of the dysplastic femur is rotational with an increase in femoral anteversion of $5^{\circ}$ to $16^{\circ}$. These authors concluded with a recommendation for the use of modular femoral components in dysplastic hips [8].

An optimal cutoff point is that point or degree wherein both the specificity and sensitivity of a diagnostic test are maximized [7]. In other words, it is the point in which a test can most accurately determine the normal from the abnormal. In our study, this point was defined as the degree at which a modular stem was used. We found that the most accurate cutoff point for determining the use of a modular stem was a femoral anteversion angle of $\geq 32^{\circ}$ meaning that patients with an estimated femoral anteversion angle, as calculated from preoperative radiographs, of $\geq 32^{\circ}$ would suggest that a modular stem be used. A request to have the stem available in the operating room at the time of surgery would be recommended. Ultimately, however, the need for the modular stem should be determined in the operating room by the surgeon.

In conclusion, we identified cutoff points for the use of modular femoral stems with standard AP and lateral preoperative plain film radiographs without the need for CT. The best cutoff value for the AP neck-shaft angle was $\geq$ $142^{\circ}$. For the lateral neck-shaft angle, the best cutoff value was $\leq 153^{\circ}$ and for femoral anteversion, it was $\geq 32^{\circ}$. When patients lie close to these cutoffs, intraoperative decisions by the surgeon are still needed to determine the best implant choice for the patient. Nevertheless, these values allow for improved preoperative planning to permit the availability of these stems when the intraoperative anteversion is $>25^{\circ}$. Future studies should evaluate the use of these cutoff points in a prospective fashion to better assess the accuracy of the measurements and correlation with intraoperative decision-making.

\section{References}

1. Biant LC, Bruce WJ, Assini JB, Walker PM, Walsh WR. Primary total hip arthroplasty in severe developmental dysplasia of the hip. Ten-year results using a cementless modular stem. $J$ Arthroplasty. 2009;24:27-32.

2. Bland JM, Altman DG. Statistical methods for assessing agreement between two methods of clinical measurement. Lancet. 1986;1:307-310.

3. Crowe JF, Mani VJ, Ranawat CS. Total hip replacement in congenital dislocation and dysplasia of the hip. J Bone Joint Surg Am. 1979;61:15-23.

4. Faldini C, Miscione MT, Chehrassan M, Acri F, Pungetti C, d'Amato M, Luciani D, Giannini S. Congenital hip dysplasia treated by total hip arthroplasty using cementless tapered stem in patients younger than 50 years old: results after 12-years followup. J Orthop Traumatol. 2011;12:213-218.

5. Harrell F, Lee K, Mark D. Tutorial in biostatistics mutlivariable prognostic models: Issues in developing models, evaluating assumptions and adequacy, and measuring and reducing errors. Stat Med. 1996;15:361-387.

6. Lin LI. A concordance correlation coefficient to evaluate reproducibility. Biometrics. 1989;45:255-268.

7. Linnet K, Brandt E. Assessing diagnostic tests once an optimal cutoff point has been selected. Clin Chem. 1986;32:1341-1346.

8. Noble PC, Kamaric E, Sugano N, Matsubara M, Harada Y, Ohzono K, Paravic V. Three-dimensional shape of the dysplastic femur: implications for THR. Clin Orthop Relat Res. 2003; 417:27-40

9. Ogata K, Goldsand EM. A simple biplanar method of measuring femoral anteversion and neck-shaft angle. J Bone Joint Surg Am. 1979;61:846-851.

10. Rosenkrantz AB, Lim RP, Haghighi M, Somberg MB, Babb JS, Taneja SS. Comparison of interreader reproducibility of the prostate imaging reporting and data system and likert scales for evaluation of multiparametric prostate MRI. AJR Am J Roentgenol. 2013;201:W612-618.

11. Royston P, Sauerbrie W. Bootstrap assessment of the stability of multivariable models. STATA J. 2009;9:547-570.

12. Sugano N, Noble PC, Kamaric E, Salama JK, Ochi T, Tullos HS. The morphology of the femur in developmental dysplasia of the hip. J Bone Joint Surg Br. 1998;80:711-719.

13. Tamegai H, Otani T, Fujii H, Kawaguchi Y, Hayama T, Marumo K. A modified S-ROM stem in primary total hip arthroplasty for developmental dysplasia of the hip. J Arthroplasty. 2013;28: 1741-1745.

14. Wiberg G. Studies on dysplastic acetabula and congenital sybluxation of the hip joint. With special reference to the complication of osteoarthritis. Acta Chir Scand Suppl. 1939;58:7-38.

15. Yiannakopoulos CK, Xenakis T, Karachalios T, Babis GC, Hartofilakidis G. Reliability and validity of the Hartofilakidis classification system of congenital hip disease in adults. Int Orthop. 2009;33:353-358. 